\title{
Deregulation of Stemness-Related Genes in Endometriotic Mesenchymal Stem Cells: Further Evidence for Self-Renewal/Differentiation Imbalance
}

\author{
Parisa Mashayekhi ${ }^{1}$, Mehrdad Noruzinia ${ }^{{ }^{*}}$ and Sepideh Khodaverdi ${ }^{2}$ \\ ${ }^{1}$ Department of Medical Genetics, Faculty of Medical Sciences, Tarbiat Modares University, \\ Tehran, Iran; ${ }^{2}$ Endometriosis Research Center, Iran University of Medical Science, Tehran, Iran
}

Received 21 August 2019; accepted 2 December 2019; published online 18 April 2020

\begin{abstract}
Background: Any irregularities in self-renewal/differentiation balance in endometriotic MSCs can change their fate and function, resulting in endometriosis development. This study aimed to evaluate the expression of OCT4 transcripts (OCT4A, OCT4B, and OCT4B1), SOX2, and NANOG in endometriotic MSCs to show their aberrant expression and to support self-renewal/differentiation imbalance in these cells. Methods: MSCs were isolated from three endometriotic and three normal endometrium samples and characterized and analyzed for the expressions of OCT4A, OCT4B, OCT4B1, SOX2, and NANOG using the qRT-PCR. Results: The expressions of OCT4 transcripts and NANOG increased significantly in endometriotic MSCs, whereas SOX2 expression did not show any significant difference. Conclusion: Our findings provide further evidence for confirming the self-renewal/ differentiation imbalance in endometriotic MSCs, as the main underlying cause of endometriosis development. This study also paves the way for further research on endometriosis treatment by focusing on endometriotic stem cells. DOI: 10.29252/ibj.24.5.328
\end{abstract}

Keywords: Endometriosis, Mesenchymal stem cells, Transcription factors

Corresponding Author: Mehrdad Noruzinia

Department of Medical Genetics, Faculty of Medical Sciences, Tarbiat Modares University, Tehran, Iran; Tel.: (+98-912) 6104835;

Fax: (+98-21) 88013030; E-mail: noruzinia@modares.ac.ir

\section{INTRODUCTION}

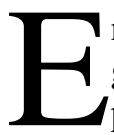
ndometriosis gynecologic presence of is a common debilitating disorder recognized by the endometrial tissue (gland and stroma) outside the uterus, especially in pelvic organs ${ }^{[1]}$. Clinically, the disease is mostly manifested with pelvic pain, painful intercourse, and infertility ${ }^{[2]}$. Retrograde menstruation is the oldest principle in endometriosis pathogenesis, and stem cell theory is the most popular issue. During each menses, stem cells are transmitted to peritoneum through retrograde menstruation ${ }^{[3]}$. Endometriosis occurs in $10 \%$ of females ${ }^{[4]}$, while retrograde menstruation appears in most women ${ }^{[5]}$; this fact highlights the different characteristics of endometriotic MSCs. Self-renewal and differentiation are essential factors in determining stem cell fate ${ }^{[6]}$, and any disturbance in self- renewal/differentiation equilibrium can alter the characteristics and functions of stem cells, thereby causing various kinds of diseases. Self-renewal/ differentiation imbalance is likely to be present in endometriotic MSCs, which could be considered as the underlying reason for endometriosis. In endometriotic women, eutopic endometrial cells have shown increased proliferation activity ${ }^{[7]}$ and decreased differentiation/decidualization capacity $^{[8]}$. Several factors, including transcription factors, epigenetic regulatory factors, and miRNAs, influence the selfrenewal/differentiation balance in stem cells ${ }^{[9]}$.

miRNAs are epigenetic factors deregulated in endometriotic MSCs ${ }^{[0]}$ and can serve as biomarkers in the early diagnosis of endometriosis ${ }^{[11]}$. Transcription factors OCT4, SOX2, and NANOG organize the core pluripotency network, which in turn regulates the pluripotency of stem cells. These factors are naturally 
expressed in both embryonic and adult stem cells ${ }^{[12,13]}$. They also participate in pathways that organize a mutual regulatory circuit with epigenetic regulatory factors like miRNAs to balance selfrenewal/proliferation and differentiation of stem cells ${ }^{[14]}$. Thus, their deregulation can disturb the stem cells balance and change their fate.

The human OCT4 gene can produce three transcripts $(O C T 4 A, O C T 4 B$, and $O C T 4 B 1)$ by alternative splicing ${ }^{[15,16]}$. OCT4A is the most studied and described isoform, and its fine-tuning is necessary for maintaining pluripotency or inducing differentiation in stem cells ${ }^{[17]}$. During the differentiation process, the expression levels of $O C T 4 A$ are decreased ${ }^{[18]}$. In tumor cell lines, OCT4B modulates $O C T 4 A$ expression as a non-coding RNA, and its overexpression enhances the expression of OCT4A and cell proliferation and decreases apoptosis ${ }^{[19]}$. Ectopic expression of $O C T 4 B$ promotes cell proliferation, migration, and angiogenesis in addition to the suppression of caspase activity $^{[20,21]}$ and its mediated differentiation ${ }^{[22]}$. $O C T 4 B 1$ is mainly expressed in pluripotent cells ${ }^{[23]}$ and is downregulated following differentiation ${ }^{[15]}$. There is a direct relationship between the expression of OCT4B1 and stemness-related genes (OCT4, SOX2, $N A N O G$, and KLF4), and its downregulation in cancer cell lines inhibits the expression of these genes ${ }^{[24]}$. OCT4B1 overexpression also accelerates cell proliferation $^{[24]}$ and has anti-apoptotic effects via suppressing caspase activity ${ }^{[25]}$. Caspase is a class of proteases involved in apoptosis, and its activity is essential for cell differentiation ${ }^{[22]}$. Fine-tuning intensity or duration of caspase signaling is vital in the death/differentiation cell fate decision ${ }^{[26]}$, therefore, $O C T 4 B$ and $O C T 4 B 1$ can influence the differentiation of stem cells by fine-tuning of the caspase activity.

It seems that the aberrant expression of stemnessrelated genes disturbs the self-renewal/differentiation balance of endometriotic MSCs in favor of increased proliferation and migration and reduced differentiation, which contributes to endometriosis development. Considering this hypothesis, we evaluated the expression levels of OCT4A, OCT4B, OCT4B1, SOX2, and $N A N O G$ in endometriotic MSCs and compared them with normal endometrial MSCs.

\section{MATERIALS AND METHODS}

\section{Specimen collection}

Human endometrial tissue samples were obtained from women aged $30-45$ years (mean $34.8 \pm 4.7$ ) in the secretory phase. Three women were healthy and volunteered for the study, and another three women underwent laparoscopic surgery for stages III and
IV endometriosis in the Rasoul Akram Hospital, Tehran, Iran. None of the women had received hormone therapy for at least three months before surgery.

\section{Isolation and culture of human endometrial MSCs}

Human endometrial tissue was isolated from the myometrium. Collected tissue was dissociated into single-cell suspensions by mechanical methods and using enzymatic digestion by collagenase type 3 (300 $\mu \mathrm{g} / \mathrm{ml}$; Sigma, Germany) at $37^{\circ} \mathrm{C}$ for 90 minutes. Endometrial stromal cells were cultured in T25 culture flasks containing DMEM/Ham's F-12 (Invitrogen, UK), $1 \%$ penicillin-streptomycin solution, and $10 \%$ FBS (Gibco, USA).

\section{Flow cytometry analysis of endometrial stromal cells}

Endometrial stromal cells in passages 3-4 were harvested and characterized using flow cytometry for cell surface markers. Cells were stained with phycoerythrin- or fluorescein isothiocyanateconjugated antibodies. Anti-human CD90, CD73, and CD146 (all from BD Bioscience, USA), as well as CD105 (Immunostep, Spain) were used as specific antibodies for MSCs and anti-human CD45 (BD Bioscience) and CD34 (Immunostep) as specific antibodies for hematopoietic stem cells, served for negative controls. Cells were analyzed using a FACS calibur apparatus (Becton Dickinson, USA), and the collected data were analyzed using FlowJo 7.6 software.

\section{Differentiation of endometrial MSCs}

To characterize, the endometrial stromal cells were seeded in 24-well plates and cultured for three weeks in osteogenic and adipogenic differentiation media, separately. Cells cultured in low-serum medium (DMEM/F12 with $1 \%$ each of FBS and antibiotic) were used as the control group. The medium was changed every 2-3 days. After three weeks, the stromal cells were fixed and stained with $4 \%$ Alizarin Red (pH 4.1) and $1 \%$ Oil Red O (both from Sigma) to assess osteogenic and adipogenic differentiation.

\section{Gene expression analyses}

Total cellular RNA was collected from cultured cells using TRIzol Reagent (Sigma). Reverse transcription of the extracted RNA was performed with the cDNA synthesis kit (Takara Bio, USA, Inc.). Specific primers for the OCT4 splice variants (OCT4A, OCT4B, and $O C T 4 B 1$ ), as shown in Table 1 , were designed as described before by Atlasi et al. ${ }^{[15]}$. Also, in order to evaluate $S O X 2, N A N O G$, and GAPDH (as an internal 
Table 1. Sequences of oligonucleotides used for real-time PCR

\begin{tabular}{ll}
\hline Primer name & Sequence $\left(\mathbf{5}^{\prime} \rightarrow \mathbf{3}^{\prime}\right)$ \\
\hline GAPDH-F & ATGAGAAGTATGACAACAGCCTC \\
CAPDH-R & CATGAGTCCTTCCACGATACC \\
OCT4A-F & CTTCTCGCCCCCTCCAGGT \\
OCT4A-R & AAATAGAACCCCCAGGGTGAGC \\
OCT4B-F & AGACTATTCCTTGGGGCCACAC \\
OCT4B-R & GGCTGAATACCTTCCCAAATAGA \\
OCT4B $1-\mathrm{F}$ & AGACTATTCCTTGGGGCCACAC \\
OCT4B1-R & CTTAGAGGGGAGATGCGGTCA \\
SOX2-F & AGTATCAGGAGTTGTCAAGGC \\
SOX2-R & CTGGGGCTCAAACTTCTCTC \\
$N A N O G-\mathrm{F}$ & CCTCTATACTAACATGAGTGTGG \\
$N A N O G-\mathrm{R}$ & CATGGAGGAAGGAAGAGGAGA \\
\hline
\end{tabular}

control) expression levels, the specific primers sets were designed using Allele ID6 and Oligo7 software (Table 1). The qRT-PCR was carried out in an AB StepOne thermocycler using the SYBR Green qPCR Master Mix (Applied Biosystems, USA), according to the manufacturer's protocol. PCR efficiency was determined using LinReg software. The expression level of each target gene was normalized in reference to the GAPDH mRNA level and analyzed using the Pfaffl method.

\section{Statistical analysis}

The data were analyzed by $t$-test using GraphPad Prism 6 software, and results with $p$ values of less than 0.05 were considered statistically significant.

\section{Ethical statement}

The above-mentioned sampling protocols were approved by the Ethics Committee of Medical Faculty of Tarbiat Modares University, Tehran, Iran (ethical code: 1395.409). Written informed consents were obtained from all the women who are participated in this study.

\section{RESULTS}

\section{Characterization of endometrial MSCs}

The expressions of mesenchymal markers CD73 (98.5\%), CD90 (99.1\%), CD105 (96.3\%), and CD146 $(84.8 \%)$ were confirmed by flow cytometry analysis. The expressions of hematopoietic markers CD34 $(0.474 \%)$ and CD45 (1.99\%) were negative in isolated cells (Fig. 1A-1H). Adipogenic and osteogenic differentiation was induced in cultured cells with specific differentiation media, then the result was visualized by Alizarin Red staining and Oil Red staining for calcium deposits and lipid vacuoles, respectively (Fig. 1I and $1 \mathrm{~J}$ ).

\section{Upregulation of $O C T 4$ transcripts in endometriotic MSCs}

Relative expressions of OCT4A showed the upregulation of this transcript $(3.58 \pm 1.11, p<0.001)$ in endometriotic MSCs (Fig. 2A). OCT4B and OCT4B1 expression levels were $2.00 \pm 0.78$ ( $p=$ $0.007)$ and $2.54 \pm 0.97(p=0.003)$ fold higher than the normal MSCs, respectively (Fig. 2B and 2C).

\section{$S O X 2$ and $N A N O G$ expressions in endometriotic MSCs \\ SOX2 expression in endometriotic MSCs did not} show any significant difference $(1.08 \pm 0.05 ; p=0.1)$ relative to the normal MSCs (Fig. 2D), but a significant increase was observed in the expression of NANOG $(2.00 \pm 0.57 ; p=0.001$; Fig. $2 \mathrm{E})$.

\section{DISCUSSION}

Stem cell theory is the most favored theory in endometriosis pathogenesis. It seems that endometriotic MSCs are different from normal ones and have impaired self-renewal/differentiation balance. The aberrant expression of transcription factors (OCT4, $S O X 2$, and $N A N O G$ ) can disturb the balance in stem cells and alter their function. Ectopic expression of these transcription factors in cancer stem cells is correlated with poor differentiation, large tumor size, and high-grade tumor ${ }^{[27]}$. Co-expression of OCT4 and $N A N O G$ in lung adenocarcinoma enhances cell proliferation and motility and decreases their differentiation by inducing cancer stem cell-like properties $^{[28]}$.

In this study, we investigated for the first time the expression of OCT4 transcripts (OCT4A, OCT4B, and $O C T 4 B 1)$, together with $S O X 2$ and $N A N O G$ in endometriotic MSCs and found that OCT4A upregulated as compared to the healthy controls. Previous studies have demonstrated the upregulation of $O C T 4 A$ in the eutopic and ectopic endometrium of women with endometriosis ${ }^{[29,30]}$. Overexpression of OCT4A mRNA enhanced self-renewal, tumorsphere generation capacity, cell motility, and invasion of medulloblastoma cells ${ }^{[31]}$. However, OCT4A decreases in stem cells during their differentiation ${ }^{[18]}$. The increased expression of $O C T 4 A$ in our study might increase self-renewal and migration in stem cells and decreas the differentiation potential.

Our findings demonstrated that $O C T 4 B$ upregulated in endometriotic MSCs. OCT4B regulated OCT4A expression by competing for endogenous RNA in a 
A
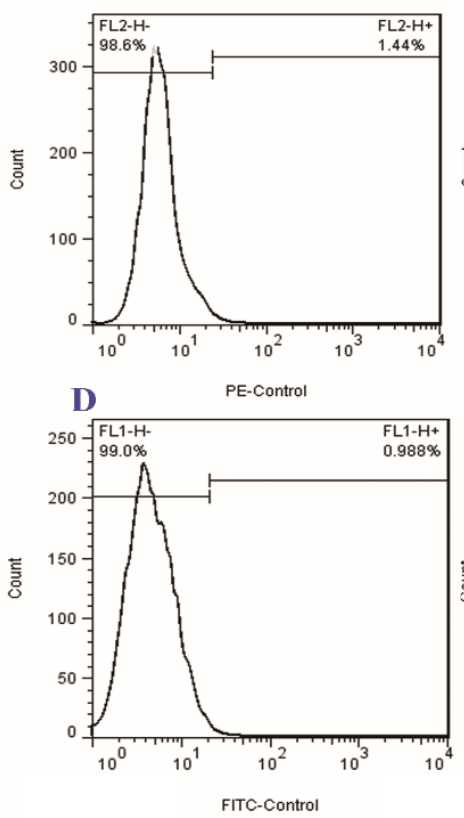

B

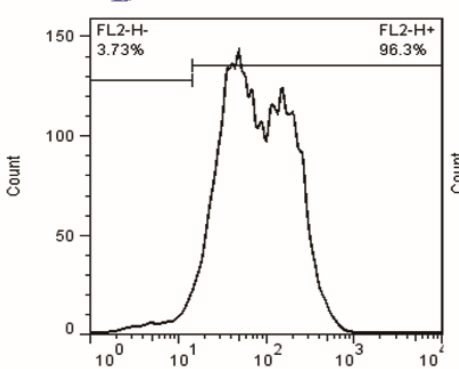

E CD105-PE

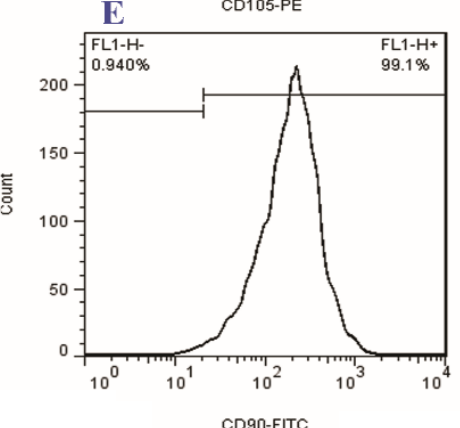

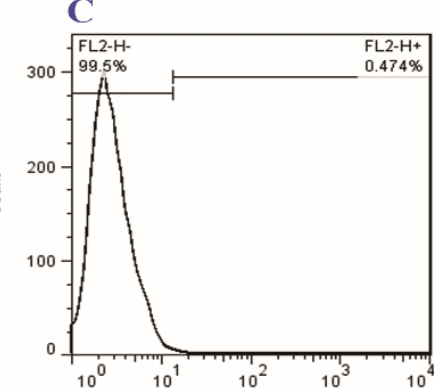

F CD34-PE

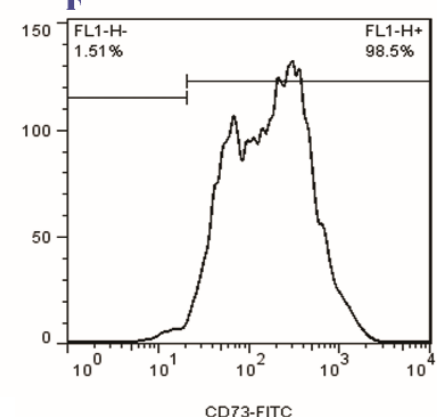

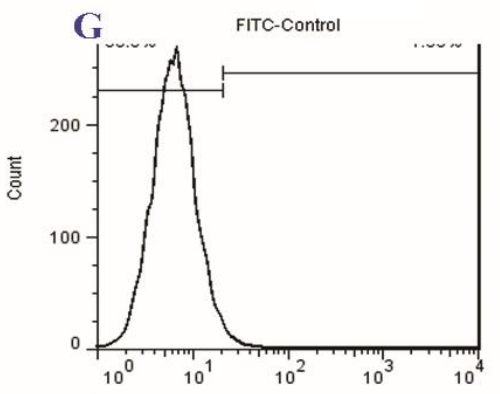

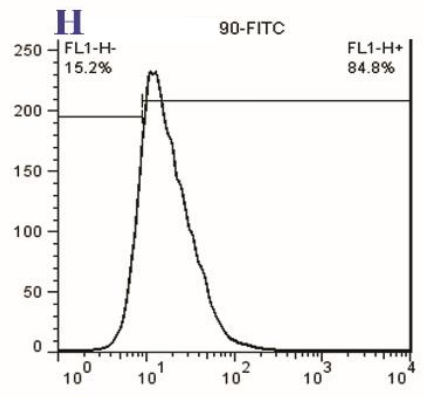

I

CD45-FITC
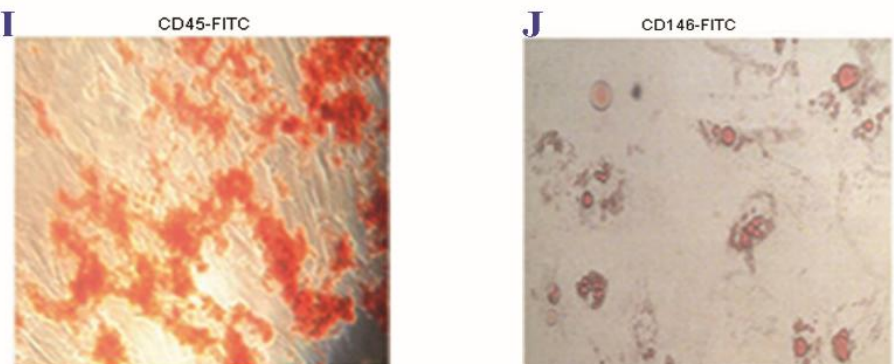

Fig. 1. Isolation and characterization of endometrial MSCs. (A) phycoerythrin-conjugated isotype control, (B) CD105 (96.3\%), (C) CD34 (0.474\%), (D) fluorescein isothiocyanate-conjugated isotype control, (E) CD90 (99.1\%), (F) CD73 (98.5\%), (G) CD45 (1.99\%), and $(\mathrm{H}) \mathrm{CD} 146(84.8 \%)$, and (I) osteogenic and (J) adipogenic differentiation of isolated endometrial MSCs.

miRNA-dependent manner in tumor cells, resulting in the increased cell proliferation and self-renewal and decreased cell apoptosis ${ }^{[19]}$. OCT $4 B$ elevates epithelialmesenchymal transition and cell migration ${ }^{[32]}$. Therefore, it is likely that the increased OCT $4 B$ expression in our study can promote self-renewal migration of stem cells and suppress caspase activity, which leads to the decreased differentiation and improved survival of migrated stem cells.

Our results suggested the increased expression of $O C T 4 B 1$ in endometriotic MSCs. OCT4B1 is in a close relationship with pluripotency regulator genes, and its downregulation causes the decreased expression of $O C T 4 A, S O X 2, N A N O G$, and KLF4 ${ }^{[24]}$. OCT4B1 enhances the proliferation and the growth of colon cancer cells by maintaining stem cell properties. Furthermore, it increases cell migration and reduces apoptosis $^{[33]}$. OCT4B1 downregulation significantly raises the activity of caspase- 3 and -7 and elevates the apoptosis rate in gastric cancer cells ${ }^{[25]}$. It seems that the increased expression of OCT4BI in this study is in favor of elevating self-renewal and the 

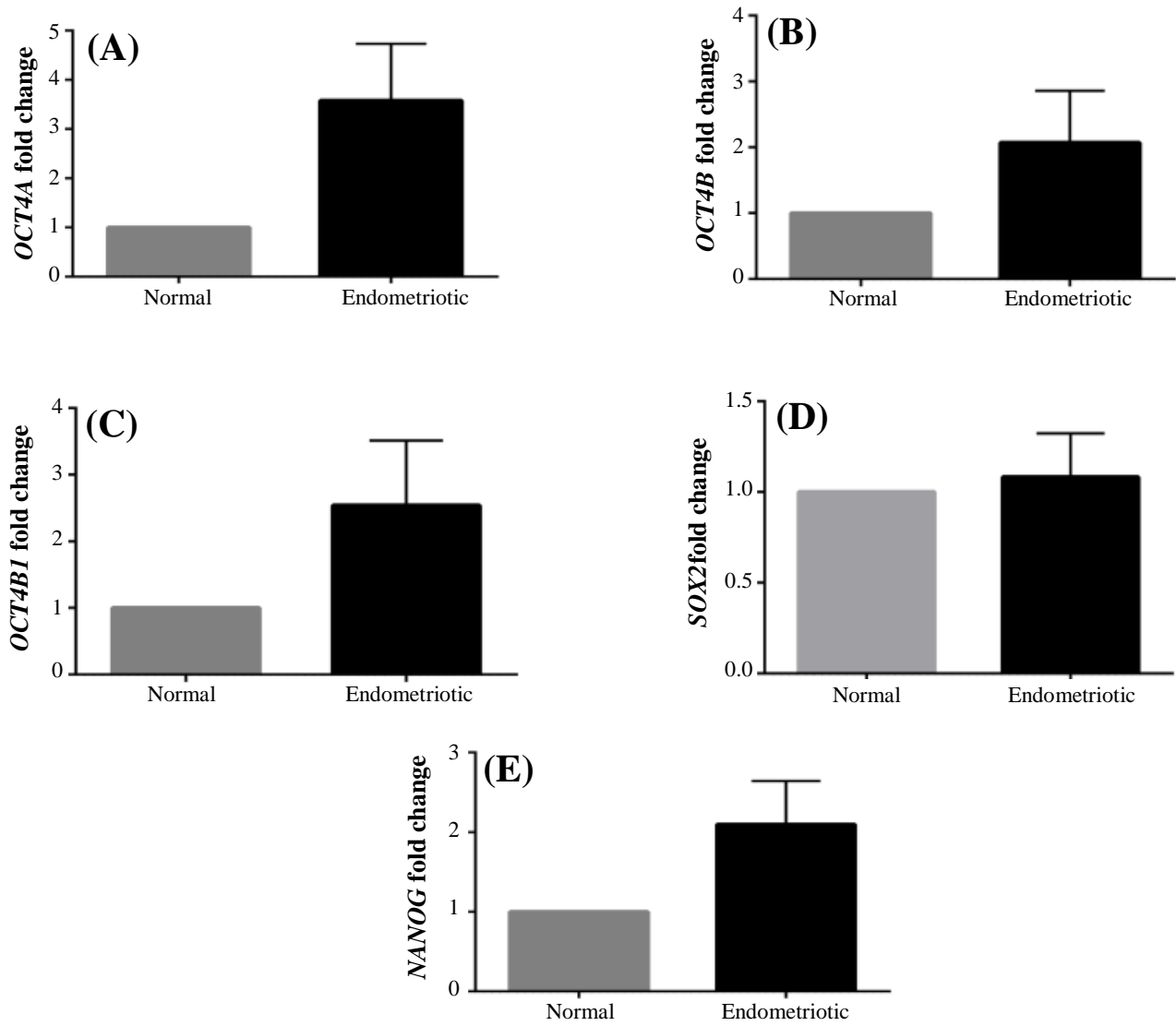

Fig. 2 Relative expressions of $O C T 4 A(\mathrm{~A}), O C T 4 B(\mathrm{~B}), O C T 4 B 1$ (C), SOX2 (D), and NANOG (E) in endometriotic MSCs in comparison with normal ones, detected by RT-PCR.

migration of endometrial MSCs in addition to decreasing their differentiation through suppressing caspase activity.

SOX2 is one of the critical transcription factors involved in stem cell fate. In this study, we did not observed any significant increase in the mRNA level of SOX2 in endometriotic MSCs.

Herein, NANOG expression raised in endometriotic MSCs. A former study also found an increase in $N A N O G$ expression in the eutopic and ectopic endometrium of endometriotic women ${ }^{[30]}$. Downregulation of $N A N O G$ in human hepatocellular carcinoma decreases the expression of $S O X 2$, OCT4, and $K L F 4$, giving rise to the reduced proliferation, invasion, and migration of cancer cells ${ }^{[34]}$. NANOG and OCT4 suppress the expression of genes that were vital for differentiation, by recruiting repressive complexes of the Polycomb-group proteins ${ }^{[35,36]}$. In our study, the increased expression of NANOG in endometriotic MSCs probably disturbs the stem cell balance by increasing the proliferation and migration and also by repressing their differentiation.

Although there is no consensus on the cause of endometriosis, evidence highlights the role of stem cells in endometriosis development ${ }^{[3]}$ and confirms their different characteristics and functions ${ }^{[8,37]}$. Our findings augmented the theory of self-renewal/ differentiation imbalance in endometriotic MSCs, which results in endometriosis development and shows their varied characteristics. Our study also helps clraify the etiology of this complex disease and pave the way for further research on endometriosis treatment by focusing on stem cells.

\section{ACKNOWLEDGMENTS}

This research was a part of a Ph.D. thesis and financially supported by the Research Deputy of Tarbiat Modares University, Tehran, Iran.

CONFLICT OF INTEREST. None declared.

\section{REFERENCES}

1. Woodward PJ, Sohaey R, Mezzetti TP, Jr. Endometriosis: radiologic-pathologic correlation. 
Radiographics 2001; 21(1): 193-216.

2. Bulletti C, Coccia ME, Battistoni S, Borini A. Endometriosis and infertility. Journal of assisted reproduction 2010; 27(8): 441-447.

3. Figueira PGM, Abrão MS, Krikun G, Taylor H. Stem cells in endometrium and their role in the pathogenesis of endometriosis. Annals of the New York academy of sciences 2011; 1221(1): 10-17.

4. Stratton P, Berkley KJ. Chronic pelvic pain and endometriosis: translational evidence of the relationship and implications. Human reproduction update 2011; 17(3): 327-346.

5. Halme J, Hammond MG, Hulka JF, Raj SG, Talbert LM. Retrograde menstruation in healthy women and in patients with endometriosis. Obstetrics and gynecology 1984; 64(2): 151-154.

6. Domen J, Weissman IL. Self-renewal, differentiation or death: regulation and manipulation of hematopoietic stem cell fate. Molecular medicine today 1999; 5(5): 201-208.

7. Park JS, Lee JH, Kim M, Chang HJ, Hwang KJ, Chang $\mathrm{KH}$. Endometrium from women with endometriosis shows increased proliferation activity. Fertility and sterility 2009; 92(4): 1246-1249.

8. Klemmt PA, Carver JG, Kennedy SH, Koninckx PR, Mardon HJ. Stromal cells from endometriotic lesions and endometrium from women with endometriosis have reduced decidualization capacity. Fertility and sterility 2006; 85(3): 564-572.

9. Olynik B, Rastegar M. The genetic and epigenetic journey of embryonic stem cells into mature neural cells. Frontiers in genetics 2012; 3: 81.

10. Mashayekhi P, Noruzinia M, Zeinali S, Khodaverdi S. Endometriotic mesenchymal stem cells epigenetic pathogenesis: deregulation of miR-200b, miR-145, and let7b in a functional imbalanced epigenetic disease. Cell journal (Yakhteh) 2019; 21(2): 179-185.

11. Bashti O, Noruzinia M, Garshasbi M, Abtahi M. miR-31 and miR-145 as potential non-invasive regulatory biomarkers in patients with endometriosis. Cell journal (Yakhteh) 2018; 20(1): 84-89.

12. Yoon DS, Kim YH, Jung HS, Paik S, Lee JW. Importance of Sox 2 in maintenance of cell proliferation and multipotency of mesenchymal stem cells in low- density culture. Cell proliferation 2011; 44(5): 428-440.

13. Huang CE, Hu FW, Yu CH, Tsai LL, Lee TH, Chou MY, Yu CC. Concurrent expression of Oct4 and Nanog maintains mesenchymal stem-like property of human dental pulp cells. International journal of molecular sciences 2014; 15(10): 18623-18639.

14. Kashyap V, Rezende NC, Scotland KB, Shaffer SM, Persson JL, Gudas LJ, Mongan NP. Regulation of stem cell pluripotency and differentiation involves a mutual regulatory circuit of the NANOG, OCT4, and SOX2 pluripotency transcription factors with polycomb repressive complexes and stem cell microRNAs. Stem cells and development 2009; 18(7): 1093-1108.

15. Atlasi Y, Mowla SJ, Ziaee SA, Gokhale PJ, Andrews PW. OCT4 spliced variants are differentially expressed in human pluripotent and nonpluripotent cells. Stem cells 2008; 26(12): 3068-3074.

16. Takeda J, Seino S, Bell GI. Human Oct3 gene family: cDNA sequences, alternative splicing, gene organization, chromosomal location, and expression at low levels in adult tissues. Nucleic acids research 1992; 20(17): 4613-4620.

17. Niwa H, Miyazaki JI, Smith AG. Quantitative expression of Oct-3/4 defines differentiation, dedifferentiation or self-renewal of ES cells. Nature genetics 2000; 24(4): 372-376.

18. Ferro F, Spelat R, D'Aurizio F, Puppato E, Pandolfi M, Beltrami AP, Cesselli D, Falini G, Beltrami CA, Curcio F. Dental pulp stem cells differentiation reveals new insights in Oct4A dynamics. PLoS one 2012; 7(7): e41774.

19. Li D, Yang ZK, Bu JY, Xu CY, Sun H, Tang JB, Lin P, Cheng W, Huang N, Cui RJ. OCT4B modulates OCT4A expression as ceRNA in tumor cells. Oncology reports 2015; 33(5): 2622-2630.

20. Li SW, Wu XL, Dong CL, Xie XY, Wu JF, Zhang X. The differential expression of OCT4 isoforms in cervical carcinoma. PLoS one 2015; 10(3): e0118033.

21. Cortes-Dericks L, Yazd EF, Mowla SJ, Schmid RA, Karoubi G. Suppression of OCT4B enhances sensitivity of lung adenocarcinoma A549 cells to cisplatin via increased apoptosis. Anticancer research 2013; 33(12): 5365-5373.

22. Fujita J, Crane AM, Souza MK, Dejosez M, Kyba M, Flavell RA, Thomson JA, Zwaka TP. Caspase activity mediates the differentiation of embryonic stem cells. Cell stem cell 2008; 2(6): 595-601.

23. Papamichos SI, Kotoula V, Tarlatzis BC, Agorastos T, Papazisis K, Lambropoulos AF. OCT4B1 isoform: the novel OCT4 alternative spliced variant as a putative marker of stemness. Molecular human reproduction Oxford academic 2009; 15(5): 269-270.

24. Hejri SJA, Mirzaei MR, Arababadi MK, Hassanshahi G, Mahmoodi M. OCT4B1 Down-regulates self-renewal genes in cancer cell lines. Pharmaceutical sciences 2014; 19(4): 117-123.

25. Asadi MH, Mowla SJ, Fathi F, Aleyasin A, Asadzadeh J, Atlasi Y, Asadi MH, Mowla SJ, Fathi F, Aleyasin A, Asadzadeh J, Atlasi Y. OCT4B1, a novel spliced variant of OCT4, is highly expressed in gastric cancer and acts as an antiapoptotic factor. International journal of cancer 2011; 128(11): 2645-2652.

26. Bell RA, Megeney LA. Evolution of caspase-mediated cell death and differentiation: twins separated at birth. Cell death and differentiation 2017; 24(8): 1359-1368.

27. You L, Guo X, Huang Y. Correlation of cancer stemcell markers OCT4, SOX2, and NANOG with clinicopathological features and prognosis in operative patients with rectal cancer. Yonsei medical journal 2018; 59(1): 35-42.

28. Chiou SH, Wang ML, Chou YT, Chen CJ, Hong CF, Hsieh WJ, Chang HT, Chen YS, Lin TW, Hsu HS. Coexpression of Oct4 and Nanog enhances malignancy in lung adenocarcinoma by inducing cancer stem celllike properties and epithelial-mesenchymal trans- 
differentiation. Cancer research 2010; 70(24): 1043310444.

29. Hwang JH, Oh JJ, Wang T, Jin YC, Lee JS, Choi JR, Lee KS, Joo JK, Lee HG. Identification of biomarkers for endometriosis in eutopic endometrial cells from patients with endometriosis using a proteomics approach. Molecular medicine reports 2013; 8(1): 183188.

30. Chang JH, Au HK, Lee WC, Chi CC, Ling TY, Wang LM, Kao SH, Huang YH, Tzeng CR. Expression of the pluripotent transcription factor OCT4 promotes cell migration in endometriosis. Fertility and sterility 2013; 99(5): 1332-1339.

31. da Silva PBG, dos Santos MCT, Rodini CO, Kaid C, Pereira MCL, Furukawa G, da Cruz DSG, Goldfeder MB, Rocha CRR, Rosenberg C, Okamoto O. High OCT4A levels drive tumorigenicity and metastatic potential of medulloblastoma cells. Oncotarget 2017; 8(12): 19192-19204.

32. Lin SC, Chung $\mathrm{CH}$, Chung $\mathrm{CH}$, Kuo $\mathrm{MH}$, Hsieh $\mathrm{CH}$, Chiu YF, Shieh YS, Chou YT, Wu CW. OCT4B mediates hypoxia-induced cancer dissemination. Oncogene 2019; 38: 1093-1105.
33. Wen KM, Zhang GH, Li J, Chen ZQ, Cheng YL, Su X, Zeng QL. OCT4B1 promotes cell growth, migration and invasion suppressing sensitivity to oxaliplatin in colon cancer. Oncology report 2015; 34(6): 2943-2952.

34. Yu AQ, Ding Y, Li CL, Yang Y, Yan SR, Li DS. TALEN-induced disruption of Nanog expression results in reduced proliferation, invasiveness and migration, increased chemosensitivity and reversal of EMT in HepG2 cells. Oncology report 2016; 35(3): 1657-1663

35. Jaenisch R, Young R. Stem cells, the molecular circuitry of pluripotency and nuclear reprogramming. Cell 2008; 132(4): 567-582.

36. Pietersen AM, van Lohuizen M. Stem cell regulation by polycomb repressors: postponing commitment. Current opinion in cell biology 2008; 20(2): 201-207.

37. Aghajanova L, Hamilton A, Kwintkiewicz J, Vo K, Giudice L. Steroidogenic enzyme and key decidualization marker dysregulation in endometrial stromal cells from women with versus without endometriosis. Biology of reproduction 2009; 80(1): $105-114$. 